\title{
A Pragmatic Justification of Deduction
}

\section{Melanie Rosen}

Whe justification of rational inference making, whether deductive or inductive, has been of interest to many philosophers. The problem of induction has concerned philosophers since the debate was first raised by David Hume. ${ }^{1}$ Hume argued that if induction is to be justified, nature must be uniform, because inductive arguments make predictions based on regularities in nature. Yet we have reasons to think that nature is not uniform. Attempts to justify the use of induction within the sciences, which began with Hume $^{2}$ who shows that we cannot use an inductive justification of induction, as this would presuppose that induction is justified, were continued by Kant in his discussion of strict universality. ${ }^{3}$ Yet an interesting point is elucidated by Carroll ${ }^{4}$ and Haack; ${ }^{5}$ that deduction is equally in need of justification despite the common assumption that deduction is inherently justified. In this paper I will focus on the attempt made by Hans Reichenbach ${ }^{6}$ to justify induction by pragmatic reasons, in reference to the inductive inferences we make on a regular basis. Other attempts have been made to show that induction is not in fact necessary within the sciences, for example this method was used by Karl Popper, ${ }^{7}$ but the fact that induction is a necessary part of human everyday reasoning means that a justification of induction is required even if induction is avoidable within the sciences. From this line of reasoning, I will show that although Reichenbach suggests that if any method of reasoning will work, induction will, this form of justification, if all else fails, can be extended to deduction. I will attempt to draw analogies between the problems of induction and problems of deduction with Carroll's paradox and Susan Haack's arguments. These analogies either strengthen a justification of induction by showing that deduction faces similar problems, or weaken our justification of deduction by showing it is not entirely justified. I will show that although

\footnotetext{
1 David Hume, Hume's Treatise of Human Nature, ed. by L. A. Selby Bigge (Oxford, Clarendon Press, 1888).

2 Immanuel Kant, The Critique of Pure Reason, trans. by Norman K. Smith (New York: Macmillan, 1978).

${ }^{3}$ Ibid.

${ }^{4}$ Lewis Carroll, "What the Tortoise Said to Achilles," in Mind, 4 (1895), 278-280. 112-119.

5 S. Haack, "The Justification of Deduction," in Mind, New Series, 85:337 (Jan., 1976),

${ }^{6} \mathrm{H}$. Reichenbach, The Rise of Scientific Philosophy (Berkeley and Los Angeles: University of California Press, 1951).

7 Karl Popper, Conjectures and Refutations: The Growth of Scientific Knowledge (London: Routledge, 1963).
} 


\section{PRAGMATIC JUSTIFICATION OF DEDUCTION}

deduction does face similar problems any justification that can work for induction will also be applicable to deduction, but not vice versa. Finally, I will show that Reichenbach's system of vindicating induction will also provide vindication for deduction, and argue that deduction preserves truth to a greater extent than induction.

\section{Induction}

Induction allows us to infer from a stock of knowledge of past observations and make a prediction of an occurrence in an unknown circumstance, either in the future or in an unobserved situation. From these predictions we also make generalisations or infer to laws of nature, stating that in all relevantly similar situations nature will be uniform. Skyrms sets out a typical inductive argument as follows:

Level 1 Inductive Argument

P1) All cases of observation $O$ have had property $P$

C) The next $\mathrm{O}$ will have $\mathrm{P}$

Or, if we are making a generalisation

$\mathrm{Ci}$ ) All instances of $\mathrm{O}$ will have $\mathrm{P}$

Skyrms refers to this as making "predictions by way of epistemic probabilities," 8 in which we observe a certain occurrence or object and speculate that it will occur the same way in the future or some other unobserved case, even in the past or somewhere else in the universe. Induction is a common method of inference, yet has shown to be ineffective in many cases, one famously described by Bertrand Russell. His example describes a chicken that uses inductive reasoning to return to the same feeding area every day but is eventually caught by the farmer, thus; inductive reasoning leads to its demise. Undoubtedly it is wrong to assume that nature is uniform in every way, or that every perceived regularity should lead us to make universal generalisations. Some regularities are temporary, as was the case in the chicken example.

According to Hume, for induction to be entirely justified, nature must be completely uniform, and the future must resemble the past. It is quite obvious that this is not the case, as nature is not entirely uniform and the future does not completely resemble the past, yet there seem to be much uniformity in nature. It is hard to imagine a completely chaotic universe that lacks regularity, but why should we assume that observed uniformities will persist? If there are some permanent uniformities in nature, how can we know which are permanent and which are not? Because we do not know if the principle of the uniformity of nature (PUN) will hold, Hume argues that trying

8 B. Skyrms, Choice and Chance: An Introduction to Inductive Logic (Belmont, California: Dickenson, 1966). 
to justify induction by saying it is useful or that it works well can be problematic. Saying that induction has been useful in the past so it will continue to be so in the future is itself an inductive inference. This is question begging as we must already assume induction to be justified. This type of higher level justification also leads to counter-intuitive consequences, such as counter induction.

Counter-inductive argument 1

P1) All observed cases of $O$ have $P$

C) The next observed case of $\mathrm{O}$ will not have $\mathrm{P}$

What could possibly justify this way of thinking? Induction has proved to be useful in the past, but counter-induction has not achieved any success. But, we can use this fact as a justification for counter induction by applying the same method we did to induction. We can establish a counter-inductive justification for counter-induction as follows.

Counter-inductive justification for counter-induction

P1) Counter induction has not provided true conclusions in the past

C) Counter-induction will provide true conclusions the next time.

Because counter induction has not worked in the past, by applying a counter-inductive inference to this, we can say that it will work in the future.

In this way, we can establish the same kind of justification for counterinduction as we did for induction, by supporting counter-induction counterinductively . Intuitively we do not expect truth preservation from counterinductive inferences, and the aforementioned justification is not convincing, but if this is the case then why should we be convinced by inductive justifications of induction? It appears that we need a separate justification for induction, otherwise we have no reason to reject counter-induction other than intuition. I think that induction is a very important tool for making predictions and the necessity of using induction in everyday life provides a justification for our continued use of such inference. It seems to me that induction is necessary to our survival, and it is hard to imagine human existence without the regular use of such inferences. Reichenbach ${ }^{9}$ argues that induction need not be completely accurate to be useful because "the conclusion of an inductive inference is not asserted [...] only posited [...] as a means to an end", this end being the prediction of future events. I will now briefly set out Reichenbach's pragmatic justification of induction, and later apply this type of justification to the problem of deduction.

${ }^{9}$ Reichenbach, The Rise of Scientific Philosophy. 


\section{PRAGMATIC JUSTIFICATION OF DEDUCTION}

\section{The Pragmatic Justification of Induction}

Although induction can notgain factual propositions to validate inductive inference by establishing PUN, we have much to gain from using induction because it can be truth preserving and provide us with predictions of future events with a good deal of accuracy, if not $100 \%$ probability. Not only is induction useful in this way, it is the best method we have for making predictions, and predicting the future is vital to our decision processes. Skyrms $\backslash$ analogy describes being forced to make a bet about the outcome of an event with the stakes being your life. . You are in a room with various light bulbs and you have to pick which colour light will turn on next. If you pick the wrong one you will be killed. You also are lead to believe that if any of the other lights turn on, a red light will also turn on. So, given no other information, what is the best choice to make? The best option, of course, is to pick red. You know if any option will work, picking red is the one that will. In the same way, the best option for making predictions is scientific induction. Skyrms argues that "it is impossible to justify the rule in question by proving that it will always, or even sometimes, yield true conclusions, given true premises[..] nevertheless, [Reichenbach claims that the] rule of induction should be adopted because one has everything to gain and nothing to lose by employing it"10 To show why this is the case, Reichenbach employs a method which describes how to establish a limiting frequency in a sequence of events. Using an options matrix, he tries to show that induction is the best option to use if sequences have limiting frequencies, and if they don't then no method will work.

The options matrix is meant to demonstrate that induction will work if anything else will. He claims that "the justification of induction is that it is the best instrument of action known to us." 11 Most of our actions are based on assumptions about what will occur in the future. I am careful not to let go of the object I am carrying because I predict that it will fall to the ground if I do, I eat my meal because I predict it would nourish me rather than poison me, and so on. Induction, according to Reichenbach, is the "best means to attain a certain aim. This aim is predicting the future" 12 Firstly I shall describe Reichenbach's method of attaining probabilities of occurrences. When witnessing a sequence of events, $\mathrm{E}$, we make observations, $\mathrm{O}$ as to particulars of the event and work out the probability of $\mathrm{O}$ occurring whenever $\mathrm{E}$ does. Let's use the example of rolling a dice, E being the event of rolling of the dice, and $\mathrm{O}$ being the observation of which number is facing up. We know there are 6 options as to how the dice could fall. As we roll the dice, we may observe the first 12 rolls land as such:

$\begin{array}{lllllllllllll}\text { Roll \# } & 1 & 2 & 3 & 4 & 5 & 6 & 7 & 8 & 9 & 10 & 11 & 12\end{array}$

Number Rolled $2 \begin{array}{llllllllllll}2 & 4 & 1 & 1 & 4 & 3 & 6 & 1 & 4 & 2 & 1 & 2\end{array}$

10 W. Salmon, "Hans Reichenbach's Vindication of Induction," in Erkenntnis, 35:1-3 (1991), 99 .

${ }^{11}$ Reichenbach, The Rise of Scientific Pbilosophy, 246.

12 Ibid. 
The frequency of rolling a 1 is $4 / 12$, a 2 is $3 / 12$ and so on. Reichenbach argues that the more events we observe, the frequency will become more accurate as we approach a limiting frequency in that sequence. In other words, our witnessed probability of an event will eventually converge with the true probability of an event. The larger the sample size, the more accurate the frequency which we have witnessed will represent the limiting frequency, until we reach a reasonable degree of accuracy. We don't expect that the frequency of rolling 1 is $4 / 12$ if it is a fair dice, but this only shows that we have not observed a sufficient number of instances of rolled dice. Salmon argues that "If the limit exists, repeated application of that rule will lead sooner or later to posits that are accurate to any desired degree of approximation"13 I expect if I roll the dice a sufficient number of times, the frequency of each number will be $1 / 6$, assuming it is a fair dice. One could argue that this would require PUN to hold if we are to attain truth preserving results. If we expect there to be regularity in the frequency of events, we already assume that there are certain regularities in the world; probabilistic regularities. This method of justification relies on limiting frequencies, a type of probabilistic regularity, for accurate results. If sequences do not have limiting frequencies, induction will not yield accurate predictions. But if the sequences do have limiting frequencies and we use inductive inference, then we will succeed in making accurate predictions. If we do not use induction, we may either fail or succeed. Alternatively, if sequences do not have limiting frequencies, then although induction will fail, any other method will fail also. Wesley Salmon sets out the matrix as follows.

Options Matrix

Induction is used Induction is not used

$\begin{array}{cc}\text { Sequence has a limit } & \text { Sequence has no limit } \\ \text { Success } & \text { Failure } \\ \text { Success or failure } & \text { Failure }\end{array}$

The table shows that if a sequence has a limit, and we use induction we will have success. Eventually observations will converge and accurately represent frequencies to make accurate predictions. There may be other systems that are successful sometimes if a sequence has a limit, but we also risk failure with these other systems. So there would be no reason not to use induction if the sequence has a limit, as it is the most successful method. But unfortunately it may be the case that not every sequence has a limit. If this is true, then induction would fail, because we need convergence to ascertain an accurate frequency, yet other systems will not work either. Despite the fact that we may rely on a version of PUN, pragmatically speaking our best bet is to use induction, because it has the best chance of success.

13 Salmon, op. cit., 102

${ }^{14}$ Ibid., 103. 


\section{PRAGMATIC JUSTIFICATION OF DEDUCTION}

This method also succeeds in providing an answer to the problem of the counter-induction. By this system, counter-induction would yield "radically incoherent sets of probability values." 15 This is because when we use induction to determine the frequency of an event, the frequency is established by observed instances of the event occurring or failing to occur, and we expect the frequency to become more accurate the more observations are made. If we tried to use counter induction as a method of ascertaining frequencies, we would not discover an accurate frequency of the event occurring. In fact, I suspect the frequencies would become more and more inaccurate, because the more times an event would occur, the more strongly counter-induction would predict that event not occurring again. There would never be a convergence of frequencies because counter-induction would predict the opposite of whatever occurrence has been regular. For example, if I used counter induction to ascertain whether the sun would rise each morning, I would continue to predict that the sun would not rise tomorrow because the frequency of the sun rising in my past observations has been 1 (or 100\%; it has occurred every time I observed) so I would expect the probability of it occurring the next time to be very low. Counter induction would fail the pragmatic justification because it is not truth preserving nor does it produce accurate predictions. Therefore we could not use a method of converging frequencies to justify counter induction.

Before I discuss the problem of deduction, I will mention two different types of justification; vindication and validation. Validation is when a rule is justified by a more fundamental principle. For example, trying to appeal to higher level principles or more fundamental rules to justify induction. This method was used by Skyrms ${ }^{16}$ to justify a particular inductive inference in reference to a higher level justification such as "inductive inferences have mostly worked in the past, so this inductive inference will also work. Some rules are considered to be the most fundamental and cannot be validated by more basic principles. The only way to justify these rules is by vindication; to show that they are truth preserving. Reichenbach's pragmatic justification is a vindication of induction in contrast to Skyrms' validation, showing that induction is vindicated by its practicality and because it is mainly truth preserving. Reichenbach would consider induction to be a fundamental principle, but whether or not it is sufficiently vindicated would be judged by how truth preserving it is. This will become important when comparing induction with deduction, which is my next task. Induction serves a purpose in granting us the ability to predict future events while most of the time preserving truth. In the same way deduction is a fundamental principle, which I will now show may face similar difficulties as induction. In this way, it may be possible to strengthen the justification of induction by putting to question a method of inference which is arguably the most reliable. Instead I will then argue that deduction is nonetheless more justifiable than induction in that any justification that can be applied to induction will further strengthen deduction.

\footnotetext{
${ }^{15}$ Ibid.

16 Skyrms, op cit.
} 


\section{Is Deduction Equally Problematic?}

\section{Carroll's Paradox}

Deductive arguments are often accepted as unproblematic as they do not require justification in the way inductive arguments do. We know that inductive arguments will not always yield true results, whereas deduction does. In deductively valid arguments, the premises always entail the conclusion. Yet Lewis Carroll argued that deduction faces similar problems to induction in that it is difficult to justify. Firstly I will describe Carroll's paradox and then show that although it does not pose a significant problem for deduction, from this arises other problems noted by Susan Haack ${ }^{17}$ which are more difficult to overcome.

Carroll's paradox sets out a simple argument of the form modus ponens. Achilles and the tortoise discuss whether we should accept the conclusion of such an argument given the truth of the premises;

(i) If $\mathrm{A}$ then $\mathrm{B}$

(ii) $\mathrm{A}$

(c) Therefore B

Any argument with this structure will always be deductively valid, and thus the truth of the conclusion is believed to be entailed by the truth of the premises. ${ }^{18}$ Achilles explains this to the tortoise who is left unconvinced. What laws governs this argument that insures that it will be valid? Why should we accept this argument? Achilles proposes that if $i$ and ii are true, c must be true. The tortoise interprets this as being a premise of the argument, thus he insists that Achilles must include this as the third premise

(i) If $\mathrm{A}$ then $\mathrm{B}$

(ii) $\mathrm{A}$

(iii) if $\mathrm{i}$ and ii are true, then $c$ must be true

(c) Therefore B

After Achilles has added this premise, the tortoise is still not convinced, and persuades Achilles to continue adding premises that state that if the previous premises are accepted, the conclusion must be accepted. This continues on until Achilles realises that there is an infinite regress to this argument. To me it does not seem obvious that we should include these extra premises which govern modus ponens as premises within the argument. Instead

${ }^{17}$ Haack, op cit.

${ }^{18}$ Some forms of logic give up on modus ponens, but I will not discuss this. Modus ponens could be substituted for any other structurally valid argument, but I will continue to use it for simplicity. 


\section{PRAGMATIC JUSTIFICATION OF DEDUCTION}

we should consider iii as a law or axiom governing the argument. If this is plausible, we would have no need for a regress of such premises. Yet, although this may solve the problem of infinite regress, we are still left with the problem of how to justify such an axiom. Why should we accept that if (i) and (ii) are true, c must be true? I will now turn to Susan Haack's discussion of this problem.

\section{Haack's Problem of Deduction}

Although I don't think that an infinite regress of premises in the justification of deduction is a real problem, if we argue that Achilles need not have added the extra premises (hence avoiding infinite regress) we end up instead with the problem of rule circularity as we saw in the first formulation of the inductive justification if induction. Susan Haack argues that because of this, deduction is in equal need of justification as induction. Firstly she describes the problems of induction and deduction as analogous. To try to justify induction deductively would be to suggest that whenever the premises are true, the conclusion must be true, which would be too strong. We know that inductive inferences are not valid, and aren't intended to be. Yet using induction to justify induction would either be circular, or end in an infinite regress of rules. Many philosophers assume that deduction need not be justified, yet if we look at this more closely, the same applies. We could either justify deduction using induction, so that if the premises are true, the conclusion is likely to be true most of the time, but this of course is too weak, as we expect deduction to be valid. Yet to justify deduction with deduction would end with the same circularity as justifying induction with induction. First I shall discuss two interpretations of the meaning of deduction that Haack set's out to get to the heart of the problem.

We could either justify deduction by definition, stating that deductive arguments are any argument in which the premises entail the conclusion, or by following a set of syntactic axioms. Both types of justification, according to Haack, are problematic. Firstly, Haack argues that if deductive arguments need no justification because by definition the premises always entail the conclusion, this leaves us with a problem: can we know for sure that any such arguments exist, and if so, which ones are they? Justifying deduction by saying it is valid by definition does not justify individual arguments, because we have not shown which arguments are the deductive ones. Secondly, if deductive arguments are valid by definition we appear to rely on circular reasoning. The argument is valid because the premises entail the conclusion, and the premises entail the conclusion because it is a valid argument. The semantic justification of deduction is therefore vacuous.

If we adopt the definition in which an argument is deductively valid (within a set rule of inference) if the conclusion is deducible from the premises by way of the axioms following the rules of inference of deduction, then we are left with a different problem; do these types of arguments always yield true result? The rules of inference can be demonstrated by setting out a truth table 
to demonstrate the truth values of different types of argument. Truth tables set out the truth values of the operators in compound propositions in deductive arguments, so these truth values are the rules of inference we must follow to ascertain the validity of an argument. But attempts to justify deduction by means of truth tables also end in circularity. For example, we could attempt to prove that modus ponens is valid by setting up a truth tree for modus ponens. A truth table tells us that the value of $A \supset B$ is 1 (or true) if the value of $A$ is 1 and the value of $\mathrm{B}$ is 1 . From this we can work out that modus ponens is a tautology; because there are no counter examples to the argument, e.g. it is not possible for the premises to be true and the conclusion false due to the rules of inference we have established for this case. If the truth table tells us that if $\mathrm{A}$ $\supset \mathrm{B}$ is true and $\mathrm{A}$ is true, then a $\mathrm{B}$ must also be true, we are relying on the rules of inference of the system of deduction to show that this argument is valid, but we have not justified the rules of inference themselves. Therefore this is a rulecircular argument because we are trying to show that modus ponens is valid, but we fall back on the very same rules of inference that we are trying to justify. We have assumed that $A \supset B \& A$ | $-B$. modus ponenș has a truth value of 1 if $A$ and $B$ both have truth values of 1 , but this rule itself is what we are trying to justify in the first place. Haack shows a counter example in which we could justify "modus morons," 19 where affirming the consequent has a truth value of 1 . If we say $\mathrm{A} \supset \mathrm{B} \& \mathrm{~B} \mid-\mathrm{A}$ affirming the consequent) is justified by the rules of inference of modus morons in which said argument has a truth value of 1 if $A$ and $B$ have a truth value of 1 , we are in a similar position as we were justifying modus ponens. So, it seems that the same justification we used for modus ponens can be employed on a very counter intuitive argument. Unlike Carroll's paradox, which suffers from premise circularity, this argument fails due to rule circularity. We assume the truth values of various arguments because of the rules of inference of the system, and this shows that the arguments are valid, but in trying to prove entailment, we presuppose that the argument is valid in setting up the rules of inference. If we change the rules of inference, we could just as easily justify counterintuitive arguments like modus morons.

\section{Inductive Justification of Deduction}

Although I agree with Haack that deduction faces problems which are analogous to the problem of induction, I will argue that whatever circularity arises from the aforementioned justifications of deduction, we are nonetheless not left in a position as problematic as induction. I will show that deduction can be vindicated pragmatically whilst avoiding some of the problems of the pragmatic justification in induction. Although we cannot justify induction by deductive means, we can have better results from justifying deduction by

${ }^{19}$ Haack, op cit., 116. 


\section{PRAGMATIC JUSTIFICATION OF DEDUCTION}

inductive means, hence deduction is not problematic to the same extent as induction.

As mentioned previously, Haack notes the difficulty in justifying induction using induction and deduction using deduction. To justify induction by means of induction not only leads to question begging, but it also allows strange systems to pass the test like counter-induction, which we have no reason to believe as being truth preserving. Alternatively, justifying deduction deductively leads either to circularity or to strange examples such as modus morons. Haack also argues that justifying induction deductively or vice versa is problematic. It seems clear that one cannot justify induction deductively but I will argue that an inductive justification of deduction, although a little strange, has the benefit of being truth preserving, hence is a preferable outcome than justifying induction deductively or induction inductively. If the vindication of deduction or by syntactic rule following or by definitional validity fail, we can fall back on a pragmatic vindication which show that deductive inferences are truth preserving. In other words, in such cases, if anything will work, deduction will. Induction cannot be justified by means of deduction because deduction is too strong. Inductive inference are not valid, thus we cannot accept this as a viable justification. Yet although induction is too weak to justify deduction, it is preferable to have a conclusion which is less bold than too bold. For example, the following argument is an inductive inference.

P1) All cases of observation $\mathrm{O}$ have had property $\mathrm{P}$

Therefore

C) The next $\mathrm{O}$ will have $\mathrm{P}$

To state that this argument is deductively valid, that given the premise we must accept the conclusion, would be a mistake. This can easily be shown by the many cases in which all observed cases have displayed property $\mathrm{P}$ but the next case does not. It may be the case that all O's we have observed have property $\mathrm{P}$, but some we have not observed do not. Therefore there is a counter example to this argument, and justifying it deductively is incorrect. Yet consider the following deductive argument;

Chain Argument:

P1) If $A$ then $B$

$\mathrm{P} 2$ ) If $\mathrm{B}$ then $\mathrm{C}$

P3) A

Therefore

C) $\mathrm{C}$

If we say that this is a strong argument, we may be making a claim that is less bold than is expected for deduction. We can justify this type of argument inductively, stating that in the past, this form of inference has yielded true results, hence it will do so in the future. Deductive arguments do not make predictions about future events, hence they do not rely on PUN, but we can 
justify the use of this type of argument, or the aforementioned chain argument in reference to perceived regularity of the reliability of the inference. We expect deductively valid arguments to be tautologies as opposed to strong arguments. But claiming that this is a strong argument is not incorrect in the way it would be incorrect to say that the inductive argument is valid. It seems we are being less bold with our estimation of the argument. Haack's aforementioned argument alludes to the fact that definitional and rule based justifications of deduction lead to circular reasoning in a similar way to Carroll's argument which highlights the infinite regress of modus ponens. But if we employ a pragmatic justification of deductive inference, we have a noncircular justification and can expect our results to be more reliable than inductive cases. One could argue that predictions and the uniformity of nature have nothing to do with deduction, but in want of a non-circular or nonvacuous justification, we can fall back on reliability based on past instances. If we say that the next use of this argument will most likely give us true results because it has always done so in the past, we are in a better position that a person trying to pass an inductive argument off as a deductive argument. Secondly, tf I were to say that this deductive argument has given true results in the past, so it will probably do the same in the future, I will have a much better success rate than a person making the same inference about induction. One could expect and 100\% success rate for such an inference, whereas a deductive justification of an inductive argument fails a-priori by intuition, or if not, then a-posteriori as soon as there is an example of said argument failing.

Yet, using induction to justify deduction brings us back to the problem of how do we justify induction? If Carroll's criticism stands, then both deduction and induction, I would argue, need to be vindicated rather than validated. We judge these to be good arguments if they are truth preserving. In Reichenbach's vindication of induction, he shows that induction is the best method of predicting unknown events, we rely on it on a day to day basis and it can be shown to be truth preserving if the sequences has a limiting frequency. If the sequence has no limiting frequency, then no method of inference will work. If we apply this to deductive arguments, we could say that all deductive arguments with a limiting frequency will yield accurate results. If we lack any other mode of justification, we could say that many observations of deductive inferences leading to true results justifies the use of such inferences. If deductive inferences do not have limiting frequencies, then they will fail, but there is no better argument method to replace them. Take modus morons. Like counter-induction, modus morons will lead to non-truth preserving results, and is thus not vindicated.

Whereas induction is necessary for predicting future events, we also rely on deductive inferences on a day to day basis. For example, a friend says they will either meet you at your place at 6 or meet you at the party at 7 . You friend is not at your house at 6 , and assuming you can believe in the truth of the disjunctive premise, it seems that you can trust they will be at the party and not at their house or at the beach. Getting by in the world would be impossible without induction, but the same can be said for deduction. Deduction could be 


\section{PRAGMATIC JUSTIFICATION OF DEDUCTION}

vindicated in the same way as induction, and to better effect. My previous experience of deductive reasoning has always been truth preserving. Hence, I can pragmatically justify deduction in that I require deductive reasoning in everyday life as much as induction. Comparing the pragmatic justification of induction with a pragmatic justification of deduction is like comparing the convergence frequency of a regular die with a die that only has ones on it. I continue to throw the die and it continues to shows ones, the same as I continue to make deductive inferences and they continue to be truth preserving. If we were to say that there is a convergence point for deductive inferences, it would be 1 , and continued observation would verify this.

A problem with the pragmatic justification of induction is that it is unclear how many cases of an event need to be observed for the event to reach its limiting frequency. This frequency may alter depending on the type of event we are observing. Certain cases may require many observations before a justified inductive inference can be made. For example, how many rolls of the dice are required before we can say that there is an equal chance of each number occurring? Occasionally, one may roll a 6 on the dice 10 times in a row. Although this is uncommon, it is a possible outcome. Then for this particular experiment, it may require hundreds or thousands of rolls before we can show that every number has an equal chance of being rolled. If this is required of a justified inductive inference, and we must be pragmatic in the same way for a deductive inference, then one would be required to observe cases of deduction in the same way. How many cases of a particular deductive inference are required before we can be justified in that inference? Most would argue that, since deduction is a-priori, none. It seems silly to assume that one must observe deductive inferences to the same extent as inductive inferences before they are vindicated. But I have ruled this a-priori justification out in the above discussion in reference to Haack. A possible method around this is to claim that deduction as a method of inference in itself has already been vindicated. Every case of deduction that has been used in the past has been valid, therefore in the future we can expect every deductive inference to also be valid. We cannot say the same for inductive inferences. Some inductive inferences have failed, such as the inference about all swans being white. Europeans believed all swans to be white because in Europe there are no black swans. This inference was disproved when black swans were observed in Australia and New Zealand. Many such cases of induction fail in the same way, therefore not all cases of induction are vindicated. We must judge inductive inferences on a case by case basis, as has been shown in the past. On the other hand, there has yet never been a failure if deduction, hence we could say that instead of judging deduction on a case by case basis, we can alternatively vindicate all deductive inferences pragmatically due to all cases being accurate in the past.

Whilst I agree with Haack that there can be analogous problems applied to deduction as well as induction, the fact that deduction is more truth preserving lends deduction to being more easily vindicated. In conclusion, I have found that induction is necessary although not guaranteed to be 
successful in all situations. It can be vindicated by the pragmatic approach, yet it is still an issue as to which sequences we should assume have limiting frequencies. Deduction on the other hand is more truth preserving than induction. It is hard to imagine a world so chaotic that inductive inferences are useless, but it is even more difficult to imagine a world where deductive arguments are not truth preserving. Therefore an attempt to strengthen induction by weakening deduction is unsuccessful. Deduction has analogous difficulties but, a pragmatic vindication for deduction is more truth preserving than one for induction.

\section{Department of Philosophy, Macquarie University, Australia}

\section{References}

Carroll, Lewis, "What the Tortoise Said to Achilles," in Mind, 4 (1895), 278280.

De Pierris, Graciela and Friedman, Michael, "Kant and Hume on Causality," in The Stanford Encyclopedia of Philosophy, $<$ http://plato.stanford.edu/archives/fall2008/entries/kant-humecausality $/>$.

Haack, S., "The Justification of Deduction," in Mind, New Series, 85:337 (Jan. 1976), 112-119.

Hume, David, Hume's Treatise of Human Nature, ed. by L. A. Selby Bigge (Oxford: Clarendon Press,1888).

Kant, Immanuel, Prolegomena to Any Future Metaphysics, trans. and ed. by Gary Hatfield (Cambridge: Cambridge University Press, 2004).

Kant, Immanuel, The Critique of Pure Reason, trans. by Norman K. Smith (New York: Macmillan, 1978).

Kelly, K., "Reichenbach, Induction and Discovery," in Erkenntnis, 35 (1991), 123-149.

Popper, K., Conjectures and Refutations: The Growth of Scientific Knowledge (London: Routledge, 1963).

Reichenbach, H., Modern Philosophy of Science (London: Routledge \& Kegan Paul, 1959).

Reichenbach, H., The Rise of Scientific Philosophy (Berkeley and Los Angeles: University of California Press, 1951)

Russell, Bertrand., The problems of philosophy (London: Oxford University Press, 1912).

Salmon, W., "Hans Reichenbach's Vindication of Induction," in Erkenntnis, 351-3 (1991), 99-122.

Salmon, W., "Should We Attempt to Justify Induction?," in Philosophical Studies (C. Sal/Tlon, Brown University, 1957).

Skyrms, B., Choice and Chance: An Introduction to Inductive Logic (Belmont, California: Dickenson, 1966). 